\title{
MicroRNA-185 downregulates androgen receptor expression in the LNCaP prostate carcinoma cell line
}

\author{
CHUNYAN LIU, ZHAOBO CHEN, XIAOYAN HU, LINA WANG, CHAOYANG LI, \\ JING XUE, PENGJU ZHANG, WEIWEN CHEN and ANLI JIANG
}

\begin{abstract}
Department of Biochemistry and Molecular Biology, Medical School of Shandong University, Jinan, Shandong 250012, P.R. China
\end{abstract}

Received April 18, 2014; Accepted January 2, 2015

DOI: $10.3892 / \mathrm{mmr} .2015 .3332$

\begin{abstract}
The present study aimed to investigate whether microRNA (miR)-185 downregulated androgen receptor expression in the LNCaP prostate carcinoma cell line. Human prostate cancer (PCa) LNCaP cells were cultured and transfected with synthetic has-miR-185 mimic or inhibitor. The transfected cells were subsequently evaluated with a viability assay, nuclear staining, reverse transcription quantitative polymerase chain reaction (RT-qPCR), dual luciferase assay and western blot analysis. The results of the western blot analysis and RT-qPCR indicated that transfection with an miR-185 mimic markedly reduced the androgen receptor (AR) protein expression levels in LNCaP cells, whereas transfection with an miR-185 inhibitor increased the protein expression of AR in the LNCaP cells. The results of the luciferase reporter assay demonstrated that the predicted target site in the AR 3' untranslated regions was a specific functional binding site for miR-185, and that AR was a direct target of miR-185. In addition, downregulation of AR by miR-185 impaired the interaction between AR and androgen response element, and downregulated the expression of the AR target gene prostate specific antigen. Data also suggested that the downregulation of AR mediated by miR-185, inhibited the proliferation and induced the apoptosis of the LNCaP cells. Therefore, the results of the present study suggested that miR-185 may be a potential negative modulator of AR-mediated signaling and may act as a tumor suppressor in prostate cancer cells.
\end{abstract}

Correspondence to: Professor Anli Jiang or Mrs Xiaoyan Hu, Department of Biochemistry and Molecular Biology, Medical School of Shandong University, 44 West Wenhua Road, Jinan, Shandong 250012, P.R. China

E-mail: zhaoch@sdu.edu.cn

E-mail: zhfe919@sdu.edu.cn

Key words: microRNA, androgen receptor, prostate cancer

\section{Introduction}

Prostate cancer ( $\mathrm{PCa}$ ) is the second most common cause of cancer-associated mortality, and the most frequently diagnosed malignancy in males in the USA $(1,2)$. Androgen receptors (AR) have the most significant functions in $\mathrm{PCa}$, in disease initiation and progression $(3,4)$. Targeting the expression of AR and inhibiting AR activity are important in androgen-dependent and androgen-independent diseases. Initially, AR is expressed by the PCa cells, which are dependent on androgens to facilitate growth (5). Common treatments involving androgen ablation have been demonstrated to temporarily alleviate the disease, however, they lead to the recurrence of highly aggressive and androgen-independent types of metastatic cancer (6,7). Previous evidence has suggested that the progression of PCa to the androgen-independent stage does not involve the loss of AR, but is induced by the restoration of AR signaling in the PCa cells (6). During the transformation from the androgen-dependent stage to the androgen-independent stage, PCa cells develop several mechanisms to facilitate the activation of AR in the androgen-depleted environment, including amplification or mutation of the AR gene, overexpression of AR co-activators and the ligand-independent activation of AR (7-9). As the development of PCa progresses, AR activity may evade regulation by ligand binding, resulting in tumor overgrowth (7). Following the activation of molecular pathways to restore AR signaling by PCa cells during the androgen-independent phase, no single therapeutic agent is able to eliminate $\mathrm{PCa}$, resulting in poor patient prognosis (10). As AR is important in the development and progression of $\mathrm{PCa}$, a strategy to downregulate the expression of AR and the inhibition of AR activity, in combination with anti-androgen therapy may prevent or delay the development of androgen-independent PCa (11-16).

Micro (mi)RNAs are short, endogenous, non-coding RNA molecules, which bind to the 3'-untranslated regions (UTRs) of target mRNAs, resulting in translational repression or message degradation (17-18). miRNAs are important in physiological cellular processes, including differentiation, proliferation and apoptosis (19-22), and have also been implicated in types of cancer $(23,24)$. Several studies have demonstrated that miRNA (miR)-185 is able to suppress 
tumor growth and progression in non-small cell lung cancer (25), ovarian, pediatric renal and breast cancer cell lines $(26,28)$, and it has been suggested that miR-185 may be important in cell proliferation.

The present study aimed to investigate whether miR-185 affected the expression of AR, cell proliferation or apoptosis of PCa LNCaP cells. The results may provide evidence for the utilization of miRNAs as novel therapeutics to target AR in $\mathrm{PCa}$.

\section{Materials and methods}

Cell culture. The human LNCaP-AD (androgen-dependent LNCaP) PCa cell line was obtained from American Type Culture Collection (ATCC; Manassas, VA, USA) and cultured in RPMI-1640 medium (HyClone, GE Healthcare Life Sciences, Little Chalfont, UK) supplemented with $10 \%$ charcoal-treated fetal bovine serum (FBS; Sigma-Aldrich, St. Louis, MO, USA), 2.05 mM L-glutamine, $100 \mathrm{U} / \mathrm{ml}$ penicillin, $100 \mu \mathrm{g} / \mathrm{ml}$ streptomycin and $10^{-8} \mathrm{~mol} / \mathrm{l} \mathrm{R} 1881$ (Sigma-Aldrich).

Androgen-independent LNCaP cells (LNCaP-AI) were generated from LNCaP-AD cells under androgen-depleted conditions. LNCAP-AI cells were produced from LNCaP-AD cells following continuous culture with phenol red-free RPMI-1640 medium (HyClone, GE Healthcare Life Sciences, Logan, UT, USA) supplemented with $10 \%$ charcoal-stripped fetal bovine serum (Serana, Bunbury, Australia) for 50 passages. The human PC-3 PCa cell line was obtained from ATCC and cultured in RPMI-1640 medium (HyClone) supplemented with $10 \%$ FBS (TBD Science, Tianjin, China), $2.05 \mathrm{mM}$ L-glutamine, $100 \mathrm{U} / \mathrm{ml}$ penicillin and $100 \mu \mathrm{g} / \mathrm{ml}$ streptomycin. All the cell lines were maintained at $37^{\circ} \mathrm{C}$ in a humidified atmosphere containing $5 \% \mathrm{CO}_{2}$.

Plasmid construction. The wild-type (WT) 3'UTR of the human AR gene was amplified from human AR complementary (c)DNA by polymerase chain reaction (PCR) using a PCR kit purchased from Takara (Dailan, China), and the fragments produced by PCR were cut using MluI and HindIII (Thermo Fisher Scientific, Waltham, MA, USA) before being cloned into the $3^{\prime}$ end of the luciferase (LUC) reporter gene in a pMIR-REPORT Luciferase vector (Ambion Life Technologies, Carlsbad, CA, USA), and named pMIR-AR-3'UTRw. The PCR primers used to amplify the AR-3'UTR were as follows: AR forward (ARF), 5'-CGACGCGTAGTCAAGCCCATCTAT-3' and reverse (ARR), 5'-CCAAGCTTGTTTGCTTGTTTTTGTT-3' (BGI, Shenzhen, China). The PCR cycling conditions were as follows: Initial denaturation at $94^{\circ} \mathrm{C}$ for $45 \mathrm{sec}$; 30 cycles of $60^{\circ} \mathrm{C}$ for $30 \mathrm{sec}$ and $72^{\circ} \mathrm{C}$ for $1 \mathrm{~min}$ and final elongation at $72^{\circ} \mathrm{C}$ for $5 \mathrm{~min}$.

Deletion mutagenesis of the putative target site for miR-185 in the WT-3'UTR of AR was performed using a two-step PCR method. Using pMIR-AR-3'UTRw as the template, a 5' flanking fragment of the deletion region was PCR amplified with the primers, ARF and deletion reverse (5'-GAAAAAGAAAAAAAGCCCAGCAAAT-3'), and the 3' lanking fragment was PCR amplified with the primers, ARR and deletion forward (5'-GCTTTTTTTCTTTTTCTTC
TTCCCTC-3') (BGI). The two fragments were linked to the primers by ligation with PCR. The ligated fragments were cloned into the MluI and HindIII sites of pMIR-REPORT Luciferase, and the plasmid was named pMIR-AR-3'UTRm.

For construction of the pGL4-androgen response element (ARE) reporter plasmid, oligonucleotides of the putative ARE were synthesized. The double-stranded ARE was generated by annealing equal quantities of forward 5'-TCGAGTGGAGGAACATATTGT ATTTATTTGGAGGAACATATTGTATTTATTA-3' and reverse 5'-AGCTTAATAAATACAATATGTTCCTCCA AATAAATACAATATGTTCCTCCAC-3' oligonucleotides at $95^{\circ} \mathrm{C}$ for $10 \mathrm{~min}$, followed by cooling to room temperature. Subsequently, the double-stranded ARE was inserted upstream of the TATA box in the pGL4.23 [luc2/minp] vector (Promega Corporation, Madison, WI, USA) to generate the recombinant plasmid, ARE-TATA box-luciferase reporter, which was named pGL4-ARE. Nucleotide sequences of all the constructs were confirmed by DNA sequencing.

Cell transfection. The LNCaP cells were transfected with the miR-185 mimic (160 nM), normal control (NC) mimic (160 nM), miR-185 inhibitor (160 nM) or NC inhibitor (160 nM) at $40 \%$ confluency using siPORT ${ }^{\mathrm{TM}} \mathrm{NeoFX}^{\mathrm{TM}}$ transfection agent (Ambion Life Technologies) at room temperature, according to the manufacturer's instructions. The pMIR-AR-3'UTRw, pMIR-AR-3'UTRm and pGL4-ARE plasmids were transfected into the cells at $90 \%$ confluency using FuGENE HD (Roche Diagnostics, Basel, Switzerland) at room temperature, according to the manufacturer's instructions.

Cell viability assay. The LNCaP-AD, LNCaP-AI and PC-3 cells were seeded into 96-well plates at $40 \%$ confluency and transfected with either the miR-185 mimic, miR-185 inhibitor, NC mimic or the $\mathrm{NC}$ inhibitor using the siPORT ${ }^{\mathrm{TM}} \mathrm{NeoFX}^{\mathrm{TM}}$ transfection agent at room temperature. The cell viabilities were then determined 24, 48, 72 and $96 \mathrm{~h}$ after transfection using an MTT assay (Sangon Biotech Co., Ltd, Shanghai, China), according to the manufacturer's instructions. Three independent experiments were performed.

Nuclear staining with Hoechst 33342. The LNCaP-AD or LNCaP-AI cells were seeded into $24-w e l l$ plates at $40 \%$ confluency and transfected with either miR-185 mimic, miR-185 inhibitor, NC mimic or NC inhibitor using siPORT ${ }^{\mathrm{TM}}$ NeoFX ${ }^{\mathrm{TM}}$ transfection agent at room temperature. Apoptotic cells were detected 48, 72 and $96 \mathrm{~h}$ after transfection using Hoechest 33258 (Beyotime Institute of Biotechnology, Shanghai, China), according to the manufacturer's instructions. Images of the stained cells were captured under a fluorescent microscope (Eclipse TE2000-U; Nikon Corp., Tokyo, Japan) at $350 \mathrm{~nm}$ excitation and $460 \mathrm{~nm}$ emission.

Reverse transcription quantitative polymerase chain reaction $(R T-q P C R)$. The LNCaP-AD or LNCaP-AI cells were seeded into 6 -well plates and transfected with either the miR-185 mimic, miR-185 inhibitor, NC mimic or NC inhibitor using siPORT ${ }^{\mathrm{TM}}$ NeoFX ${ }^{\mathrm{TM}}$ transfection agent. The total RNA was extracted $72 \mathrm{~h}$ after transfection using TRIzol reagent (Invitrogen Life Technologies, Carlsbad, CA, USA). 
First strand cDNA was synthesized from $10 \mathrm{ng}$ total RNA using gene specific primers. RT-qPCR was performed using MonsterScript ${ }^{\mathrm{TM}}$ Reverse Transcriptase (Epicentre, Illumina, Inc., Madison, WI, USA) and SYBR ${ }^{\circledR}$ Green I nucleic acid gel stain (Molecular Probes Life Technologies, Carlsbad, CA, USA).

The primers used were as follows: miR-185, forward 5'-GGTGGAGAGAAAGGCAGT-3' and reverse 5'-CAGTGCGTGTCGTGGAG-3'; U6 snRNA, forward 5'-GCTTCGGCAGCACATATACTAAAAT-3' and reverse 5'-CGCTTCACGAATTTGCGTGTCAT-3'. U6 snRNA was used as a control gene for normalization. The relative quantity of miR-185 was determined using the $2^{-\Delta \Delta C t}$ method (29).

The primers used for AR were: Forward 5'-СТТСССТСССТ АТCТААССCTC-3' and reverse 5'-TCTAAAC TTCCCGTGGCATAA-3'; for PSA were: Forward 5'-AGGTGTGCTGACTATGTGGTGAC-3' and reverse 5'-GGTTGAGGTTCCAGGTGCTT-3'; and for GAPDH were: Forward 5'-GGGAAACTGTGGCGTGAT-3' and reverse 5'-GAGTGGGTGTCGCTGTTGA-3'. PCR conditions for $\mathrm{AR}$ were: initial denaturation at $95^{\circ} \mathrm{C}$ for $5 \mathrm{~min} ; 35$ cycles of $95^{\circ} \mathrm{C}$ for $10 \mathrm{sec}, 59^{\circ} \mathrm{C}$ for $15 \mathrm{sec}, 72^{\circ} \mathrm{C}$ for $20 \mathrm{sec}$ and $82^{\circ} \mathrm{C}$ (fluorescence collection) for $5 \mathrm{sec}$. PCR conditions for PSA and GAPDH were: initial denaturation at $95^{\circ} \mathrm{C}$ for $5 \mathrm{~min} ; 35$ cycles of $95^{\circ} \mathrm{C}$ for $10 \mathrm{sec}, 59^{\circ} \mathrm{C}$ for $15 \mathrm{sec}, 72^{\circ} \mathrm{C}$ for $20 \mathrm{sec}$ and $80^{\circ} \mathrm{C}$ (fluorescence collection) for $15 \mathrm{sec}$. GAPDH was used as a control for normalization, and the data were analyzed with Rotor-Gene Real-Time Analysis software 6.0 (Corbett Research, Mortlake, Australia) according to the standard curve.

Dual-luciferase assay. The LNCaP-AD cells were seeded in 24-well plates and transfected for $24 \mathrm{~h}$ with either the miR-185 mimic, miR-185 inhibitor, NC mimic or NC inhibitor using siPORT ${ }^{\mathrm{TM}} \mathrm{NeoFX} \mathrm{FM}^{\mathrm{TM}}$ transfection agent. Subsequently, the LNCaP-AD cells were co-transfected with either pMIR-AR-3'UTRw $(0.3 \mu \mathrm{g})$, pMIR-AR-3'UTRm $(0.3 \mu \mathrm{g})$ or pMIR-REPORT $(0.3 \mu \mathrm{g})$, or co-transfected with pGL4-ARE $(0.3 \mu \mathrm{g})$ or pGL4.23[luc2/minp] using FuGENE HD transfection reagent (Roche Diagnostics, Mannheim, Germany) for $48 \mathrm{~h}$. The activities of firefly (M1) and Renilla (M2) luciferase were measured using a Dual Luciferase Assay system (Promega Corporation), according to the manufacturer's instructions. The transfection efficiency was normalized to the pRL-TK control vector $(0.04 \mu \mathrm{g}$; Promega Corporation). Three independent experiments were performed in duplicates.

Western blot analysis. The LNCaP-AD or LNCaP-AI cells were seeded into 6-well plates and transfected with either the miR-185 mimic, miR-185 inhibitor, NC mimic or NC inhibitor using siPORT ${ }^{\mathrm{TM}}$ NeoFX ${ }^{\mathrm{TM}}$ transfection agent. The cells were harvested 48 and $72 \mathrm{~h}$ after transfection for protein extraction. The protein $(20 \mu \mathrm{g})$ was resolved on an 10\% SDS-PAGE gel and electrotransferred onto nitrocellulose membranes (Bio-Rad Laboratories, Inc., Hercules, CA, USA) using a DYCZ-40D (Beijing Liuyi Instrument Factory, Beijing, China). Following blocking with $5 \%$ milk in Tris-buffered saline with $0.05 \%$ Tween-20 (TBST) buffer (Sangon Biotech Co., Ltd) and washing with TBST buffer, the membrane was incubated with mouse monoclonal anti-human AR (1:1,000; BD Biosciences,
Franklin Lakes, NJ, USA) or mouse anti- $\beta$-actin (1:1,000; Santa Cruz Biotechnology, Inc., Dallas, TX, USA) at $4^{\circ} \mathrm{C}$ for $12 \mathrm{~h}$, followed by incubation with horseradish peroxidase-labeled goat anti-mouse polyclonal secondary antibody (1:2,000; Zhongshan Golden Bridge Biological Technology, Beijing, China) for $1 \mathrm{~h}$ at room temperature. The immunoreactive bands were visualized by enhanced chemiluminescence (Santa Cruz Biotechnology, Inc.). The protein expression levels of AR in each sample were determined by normalizing AR band intensity to that of $\beta$-actin.

Sequence Analysis. TargetScan predicts the biological targets of miRNAs. This is via searching a database for conserved 8 mer and 7 mer sites, which match seed regions of the miRNA being screened, nonconserved sites are also predicted. In addition sites with mismatches in the seed region that are compensated by conserved 3' pairing are also identified. In mammals, the predictions are ranked based on the predicted targeting efficacy as calculated using context+ scores of the sites. The predictions are also ranked by their probability of conserved targeting. Conserved targeting has also been detected within open reading frames.

\section{Results}

miR-185 downregulates protein expression levels of $A R$ in LNCAP cells. To investigate the potential effects of miR-185 on the regulation of AR expression, LNCaP-AD and LNCaP-AI cells were transfected with an miR-185 mimic or inhibitor for 48 and $72 \mathrm{~h}$, and the expression levels of AR were detected using western blot and qPCR analyses. The results indicated that transfection with the miR-185 mimic markedly reduced the protein levels of $A R$, while transfection with the miR-185 inhibitor increased the protein levels of AR in the LNCaP-AD (Fig. 1A) and LNCaP-AI (Fig. 1B) cells.

The mature miR-185 levels in the miR-185-transfected LNCaP cells were measured by qPCR. The mature levels of miR-185 in the LNCaP cells transfected with the miR-185 mimic were significantly higher compared with those in the NC mimic-treated cells (Fig.1C), which indicated that the synthetic miR-185 mimic was effectively transfected and matured in the LNCaP cells. However, differences in the mRNA expression levels of AR were not detected in all types of transfected LNCaP cells (Fig. 1D). The results demonstrated that miR-185 reduced the protein levels of AR by inducing translational repression, but not AR mRNA degradation.

miR-185 interacts with the putative target site in the AR-3'UTR. Sequence analysis, using the TargetScan tool (version 5.2; http://www.targetscan.org/vert_50/), indicated that a putative binding site for miR-185 was located at 173-179 bp of the AR-3' UTR. To assess whether miR-185 interacted with this predicted binding site in the AR-3' UTR to reduce the expression of AR, wild-type 3'UTR (3'UTRw) and the 3'UTR of the AR gene, containing deletion mutations (3'UTRm), were cloned and inserted downstream of the luciferase gene in a reporter plasmid (pMIR-REPORT Luciferase), and named pMIR-AR-3'UTRw and pMIR-AR-3'UTRm. Each of these constructs were cotransfected into the LNCaP-AD cells with either the miR-185 mimic, miR-185 inhibitor, NC mimic or 

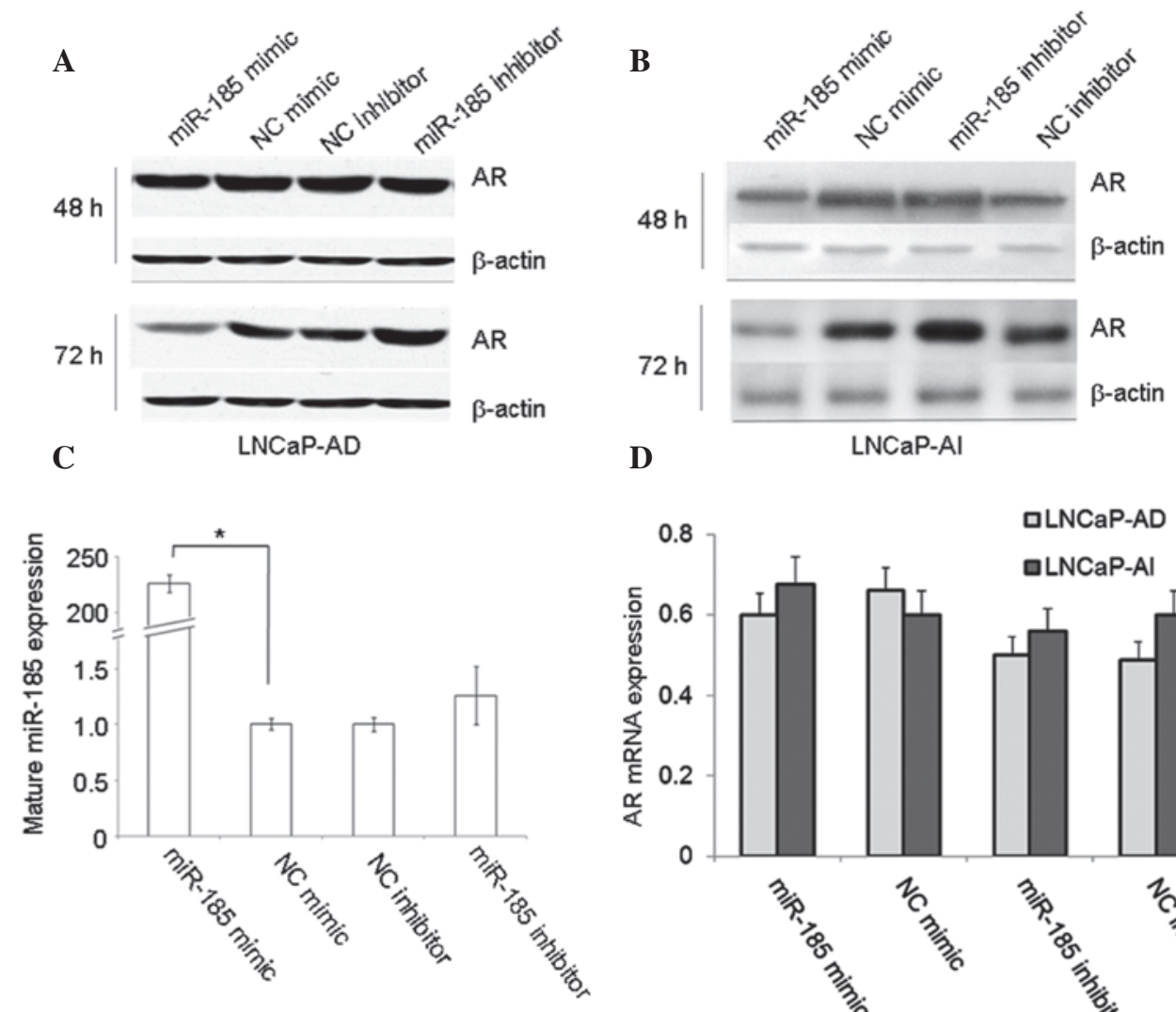

D

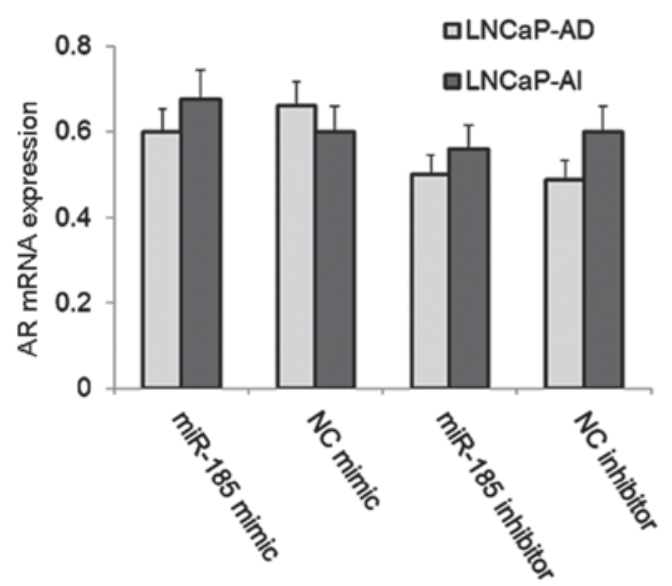

Figure 1. miR-185 targets the expression of AR in LNCaP cells. LNCaP-AD and LNCaP-AI cells were transfected with $160 \mathrm{nM}$ miR-185 mimic, miR-185 inhibitor, $\mathrm{NC}$ mimic or $\mathrm{NC}$ inhibitor for 48 and $72 \mathrm{~h}$. Western blot analysis was performed to detect the effects of miR-185 on the protein expression of AR in the (A) LNCaP-AD and (B) LNCaP-AI cells. $\beta$-actin was used as a loading control. (C) Expression of mature miR-185 in the miR-185-transfected LNCaP cells. U6 small nuclear RNA or GAPDH was used as the internal control for qPCR analysis. qPCR was performed to evaluate the effects of miR-185 on the mRNA expression levels of AR $72 \mathrm{~h}$ after transfection in the (D) LNCaP-AD and LNCaP-AI cells. Values are expressed as the mean \pm standard deviation. Experiments were repeated more than three times. "P<0.05. miR-185, microRNA-185; AR, androgen receptor; AD, androgen-dependent; AI, androgen independent; NC, normal control; qPCR, quantitative polymerase chain reaction.

A AR mRNA (NM_000044)

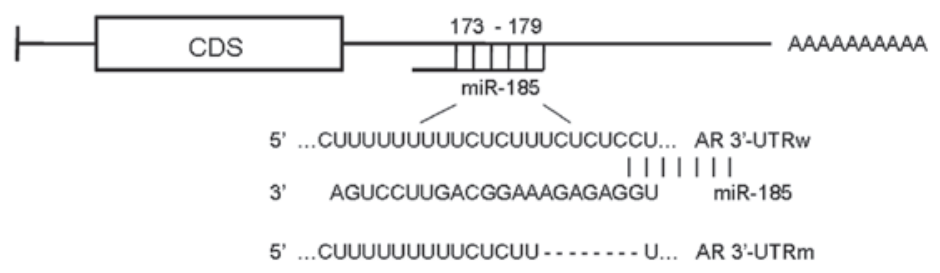

B

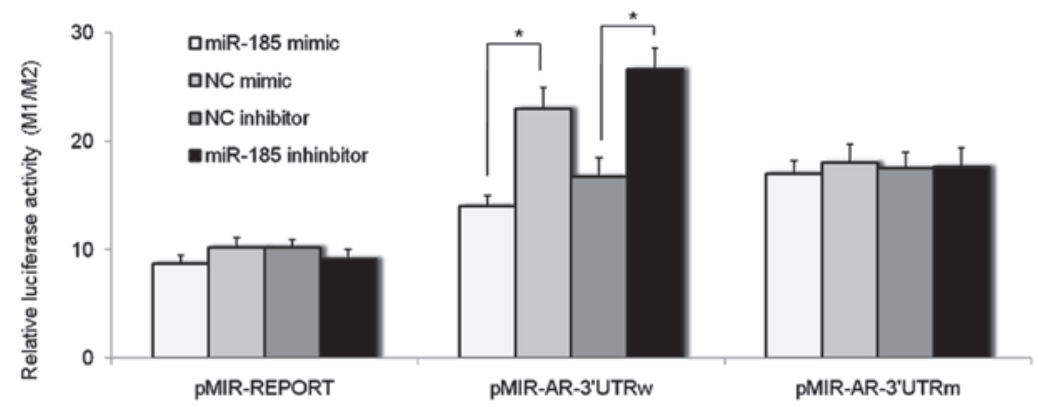

Figure 2. miR-185 directly targets the AR-3'UTR. (A) Schematic representation of AR mRNA, indicating the positions and sequences of the predicted miR-185 binding sites located in the AR-3'UTR. (B) LNCaP cells were transfected with $160 \mathrm{nM}$ miR-185 mimic, miR-185 inhibitor, NC mimic or NC inhibitor for $48 \mathrm{~h}$, and subsequently cotransfected with pMIR-AR-3'UTRw or pMIR-AR-3'UTRm for another $48 \mathrm{~h}$. Luciferase activity was detected using a Dual Luciferase Assay system and was plotted as the ratio of firefly (M1) to Renilla (M2) luciferase activity (M1/M2). Data are expressed as the mean \pm standard deviation of six individual values. Experiments were repeated more than three times. " $\mathrm{P}<0.05$. miR, microRNA; AR, androgen receptor; 3 'UTR, $3^{\prime}$ untranslated region; NC, normal control; pMIR-REPORT, luciferase reporter gene plasmid; pMIR-AR-3'UTRw, plasmid containing wild-type 3'UTR of human AR gene; pMIR-AR-3'UTRm, plasmid containing mutant 3'UTR of human AR gene. 
A

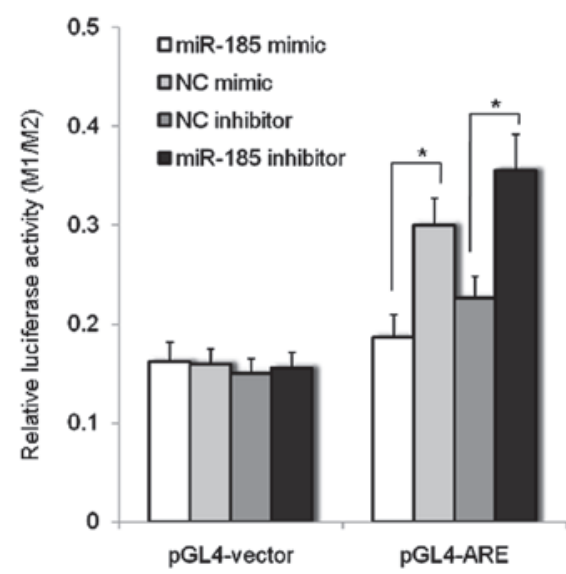

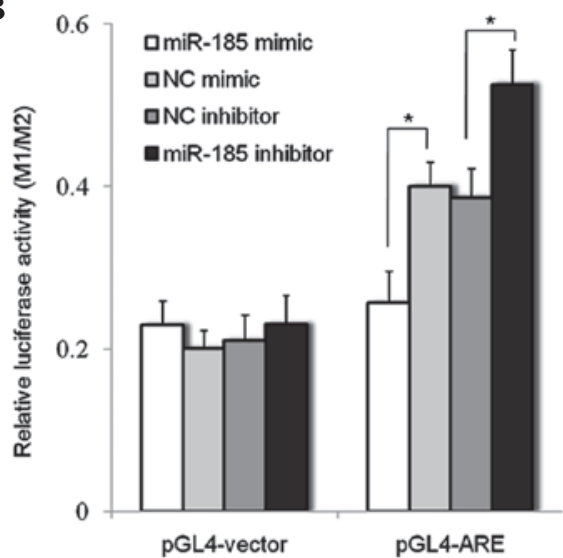

Figure 3. miR-185 impairs the interaction between AR and ARE. (A) LNCaP-AD and (B) LNCaP-AI cells were transfected with miR-185 mimic, miR-185 inhibitor, NC mimic or NC inhibitor for $48 \mathrm{~h}$, and subsequently cotransfected with the pGL4-ARE or pGL4 vector for another $48 \mathrm{~h}$. Luciferase activity was evaluated using a Dual Luciferase Assay system and was plotted as the ratio of firefly (M1) to Renilla (M2) luciferase activity (M1/M2). Data are expressed as the mean \pm standard deviation of six individual values. Experiments were repeated more than three times. ${ }^{*} \mathrm{P}<0.05$. AR, androgen receptor; ARE, androgen response element; AD, androgen dependent; AI, androgen independent; miR, microRNA; NC, normal control; pGL4-ARE, pGL4 ARE reporter plasmid; pGL4 vector, control plasmid.

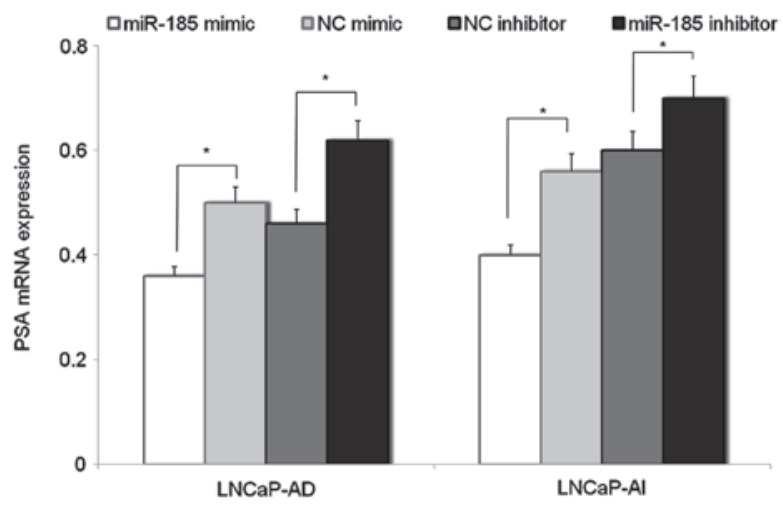

Figure 4. miR-185 downregulates the mRNA expression of PSA. LNCaP-AD and LNCaP-AI cells were transfected with miR-185 mimic, miR-185 inhibitor, $\mathrm{NC}$ mimic or NC inhibitor, and the cells were harvested $72 \mathrm{~h}$ after transfection. Total RNA was extracted and a quantitative polymerase chain reaction was performed to detect the effects of miR-185 on the mRNA expression of PSA. GAPDH was used as a control for normalization, and the relative mRNA expression of PSA was relative to that of GAPDH. Data are expressed as the mean \pm standard deviation of three individual values. Experiments were repeated more than three times. "P<0.05. miR, microRNA; PSA, prostate specific antigen; AD, androgen dependent; AI, androgen independent; NC, normal control.

A

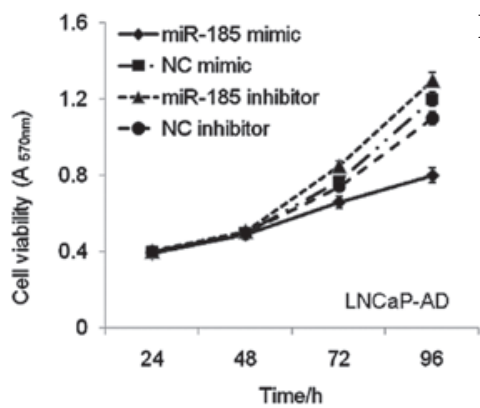

B

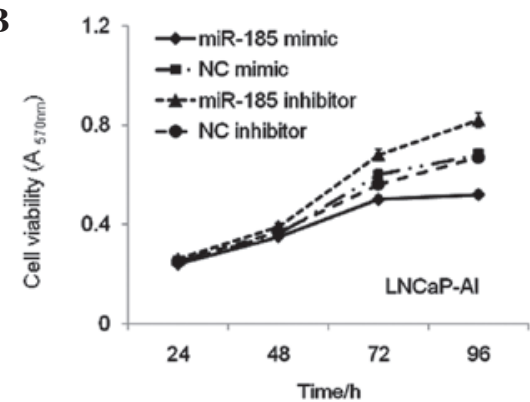

C

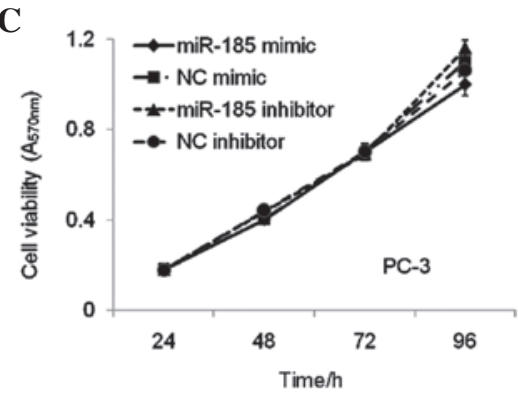

Figure 5. miR-185 inhibits the growth of LNCaP cells. (A) LNCaP-AD, (B) LNCaP-AI and (C) PC-3 cells were transfected with miR-185 mimic, miR-185 inhibitor, NC mimic or NC inhibitor. Cell viability was detected 24, 48, 72 and $96 \mathrm{~h}$ after transfection using an MTT assay. Data are expressed as the mean \pm standard deviation of six individual values. miR, microRNA; AD, androgen dependent; AI, androgen independent; $\mathrm{NC}$, normal control.

NC inhibitor. Luciferase activity was measured $48 \mathrm{~h}$ after transfection. The results (Fig. 2) indicated that transfection with the miR-185 mimic markedly reduced luciferase activity, while the miR-185 inhibitor enhanced luciferase activity of the pMIR-AR-3'UTRw. However, neither the miR-185 mimic nor miR-185 inhibitor altered the luciferase activities of the pMIR-AR-3'UTRm or pMIR-Report plasmids. Therefore, these data indicated that the predicted target site in the AR 
A

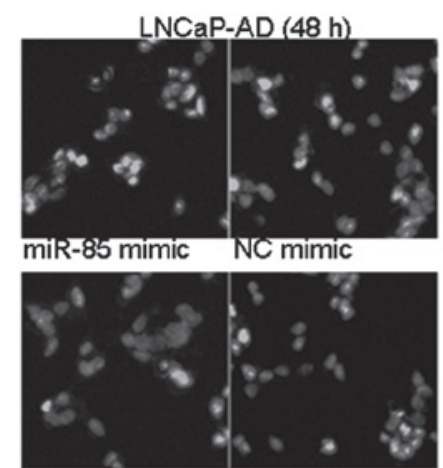

miR-185 inhibitor NC inhibitor

B

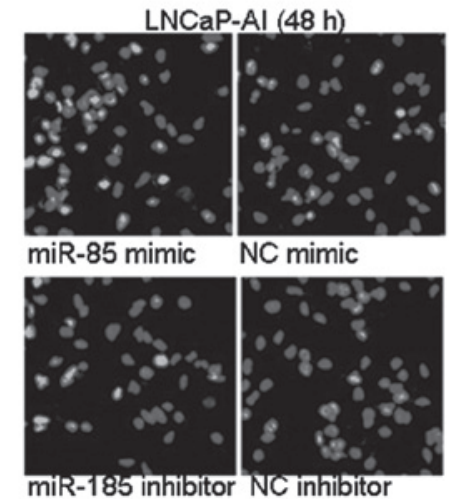

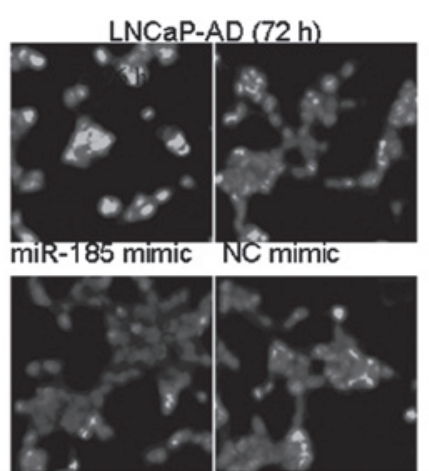

miR-185 inhibitor NC inhibitor

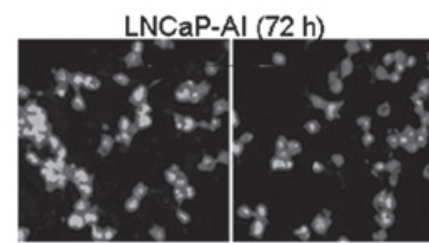

miR-185 mimic NC mimic

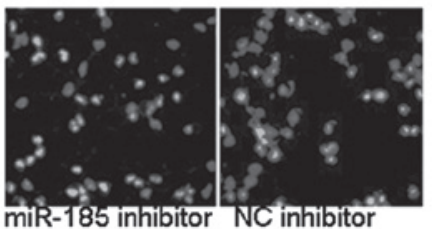

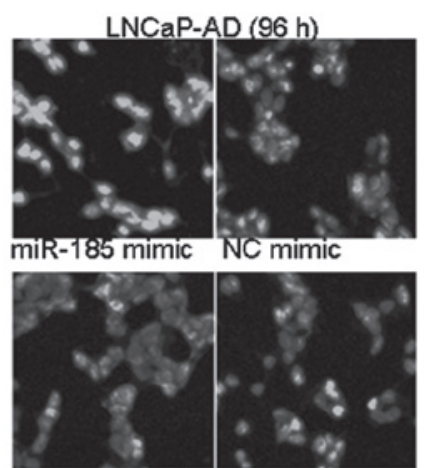

miR-185 inhibitor NC inhibitor

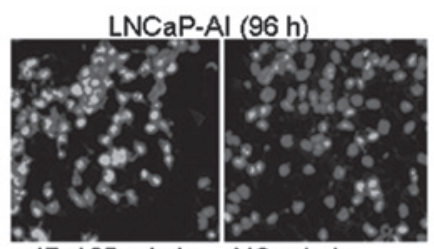

miR-185 mimic NC mimic

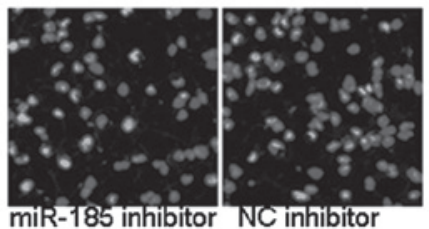

Figure 6. miR-185 induces apoptosis in LNCaP cells. The apoptosis of (A) LNCaP-AD and (B) LNCaP-AI cells was determined 48,72 and 96 h following transfection with $160 \mathrm{nM}$ miR-185 mimic, miR-185 inhibitor, NC mimic or NC inhibitor by nuclear staining with Hoechst 33342 . Images of the stained cells were captured under a fluorescent microscope at $350 \mathrm{~nm}$ stimulation and $460 \mathrm{~nm}$ emission. (magnification, x100). miR, microRNA; NC, normal control; AD, androgen dependent; AI, androgen independent.

3'UTR was a specific functional binding site for miR-185, and that AR was a direct target of miR-185.

miR-185 impairs the interaction of AR with ARE. AR is a ligand-activated transcription factor, which induces the transcription of genes exhibiting ARE in their regulatory regions (3). In order to examine whether the downregulation of AR by miR-185 impaired the interaction between AR and ARE, an ARE sequence was synthesized and inserted upstream of the TATA box in pGL4.23 [luc2/minp] plasmids, named pGL4-ARE. pGL4-ARE was cotransfected into the LNCaP-AD and LNCaP-AI cells with either the miR-185 mimic or inhibitor. Luciferase activity was measured $72 \mathrm{~h}$ after transfection. The results indicated that transfection with the miR-185 mimic reduced luciferase activity, while miR-185 inhibitor increased the luciferase activity of pGL4-ARE in LNCaP-AD (Fig. 3A) and LNCaP-AI (Fig. 3B) cells, demonstrating that downregulation of AR by miR-185 may impair the interaction between AR and ARE.

miR-185 down-regulates the expression of AR target gene prostate specific antigen (PSA). PSA is a prostate specific gene, regulated by AR. In the present study, the effects of AR downregulation by miR-185 on the expression of PSA mRNA in LNCaP cells was evaluated. The results of PCR analysis indicated that the expression of PSA mRNA in LNCaP-AD and LNCaP-AI cells transfected with miR-185 mimic was reduced compared with that in cells transfected with the NC mimic, while the mRNA expression of PSA in the miR-185 inhibitor-transfected LNCaP cells was increased compared with the NC inhibitor-transfected cells (Fig. 4).

miR-185 inhibits the proliferation of LNCaP cells. To investigate the potential of miR-185 to inhibit the growth of LNCaP cells by downregulation the expression of AR, the LNCaP-AD, LNCaP-AI and PC-3 cells were transfected with either the miR-185 mimic or inhibitor, and the numbers of viable cells were measured 24, 48, 72 and $96 \mathrm{~h}$ after transfection using an MTT assay. The results indicated that the miR-185 mimic reduced, while the miR-185 inhibitor increased the numbers of viable LNCaP-AD (Fig. 5A) and LNCaP-AI (Fig. 5B) cells. The same transfection procedure was performed in PC-3 cells, not expressing AR, in which the miR-185 mimic or inhibitor had no significant effects on cell proliferation (Fig. 5C). These data demonstrated that the effect of miR-185 on cell growth was associated with downregulation of $\mathrm{AR}$ in the $\mathrm{LNCaP}$ cells.

miR-185 induces the apoptosis of LNCaP cells. The LNCaP-AD and LNCaP-AI cells were transfected with either miR-185 mimic or inhibitor for 48, 72 and $96 \mathrm{~h}$. Apoptosis was assessed using Hoechest 33258 staining. As shown in Fig. 6, following transfection with miR-185 mimic, significantly more LNCaP-AD and LNCaP-AI cells exhibited chromatin condensation and marginalization compared with the NC mimic-transfected cells. By contrast, transfection with the miR-185 inhibitor resulted in a decreased number of LNCaP-AD and LNCaP-AI cells with nucleic chromatin condensation compared with the cells transfected with the NC 
inhibitor. These data demonstrated that the miR-185-mediated downregulation of AR induced apoptosis of the LNCaP cells.

\section{Discussion}

In the past few years, numerous studies have demonstrated that miRNAs are important in the developmental process of cancer (30-33). They may function as oncogenes or tumor suppressor genes (34) in tumorigenesis, and regulate multiple cellular processes involved in the progression of cancer. Due to each miRNA regulating numerous potential targets, identification of the true target genes that are involved in cancer cell behaviors is important for understanding the mechanisms underlying the functional contributions of miRNAs in tumor development and progression.

Several studies have demonstrated that miR-185 functions as a tumor suppressor, which is able to suppress tumor growth and progression in non-small lung carcinoma, ovarian, pediatric renal and breast cancer cell lines (25-28). It has also been suggested that miR-185 may be important in cell proliferation (25-28). However, the mechanisms by which miR-185 inhibits cell proliferation in different cancer cells vary. miR-185 suppresses the growth of the human non-small cell lung cancer cell lines and induces cell cycle arrest by suppressing the mRNA expression of cell cycle regulating genes, including CDK6 and AKT1 (25). Additionally, miR-185 suppresses tumor growth and progression by targeting the Six 1 oncogene in multiple types of human cancer, including pediatric renal tumors, aggressive ovarian cancer and breast cancer (26). In colorectal cancer cells, miR-185 directly regulates the expression of RhoA and $\mathrm{Cdc} 42$ and their associated functions, including proliferation and invasion (35). In contrast to its role in tumor suppression, miR-185 was also demonstrated to induce tumor growth and progression in clear cell renal cell carcinoma (36). The expression of miR-185 was inversely correlated with its putative target PTPN13, which suppressed cell growth and induced apoptosis as a tumor suppressor gene by inhibiting phosphoinositide 3-kinase (PI3K)/AKT signaling (37). These findings suggest that miR-185 may target differing genes in various cell types, which contribute to different biological processes.

The results of the present study confirmed miR-185 as a tumor suppressor gene, which exerted its effect through modulating the expression of AR, inhibiting cell proliferation and enhancing apoptosis of the LNCaP cells. Computational analysis revealed a potential binding site for miR-185 in the 3'UTR of AR. Deletion mutagenesis and a luciferase-based reporter gene assay demonstrated that the predicted miR-185 target sites in the AR 3'UTR were functional. Furthermore, data indicated that miR-185 effectively downregulated the protein expression of AR, impaired the interaction of AR with ARE and downregulated the expression of the AR target gene, PSA.

$\mathrm{AR}$ is a ligand-dependent transcription factor belonging to the nuclear hormone receptor superfamily (38). AR functions as a ligand-dependent transcription factor by binding to AREs in the regulatory regions of specific androgen-regulated target genes (39). The development of PCa and growth of prostate tissue depend on androgen signaling via the
AR $(14,40)$; therefore, downregulation of the expression levels of AR and androgens is a basic therapeutic strategy in preventing the development of $\mathrm{PCa}$.

Based on the significance of AR for the androgen-dependent and androgen-independent growth of PCa cells (6), understanding the association between miR-185 and AR is important for investigation of prostate carcinogenesis and therapeutics. An increasing number of studies have focused on improving PCa treatment by developing more effective strategies for silencing AR (41-44). A combinatorial approach, involving the simultaneous targeting of multiple pathways, is an accepted approach in the development of PCa therapy and the development of numerous therapeutic options, which can be evaluated in combinatorial therapy settings is being emphasized at present (10). Therefore, the results of the present study, revealing that miR-185 mediated the repression of AR expression and activity, are of significance.

In conclusion, the present study demonstrated that miR-185 directly targeted the AR-3'UTR, to inhibit the expression of $\mathrm{AR}$, and acted as a tumor suppressor in the PCa cells. miR-185 is, therefore, a potential negative modulator of AR-mediated signaling with potential for use in $\mathrm{PCa}$ therapeutics.

\section{Acknowledgements}

This study was supported by grants from the National Natural Science Foundation of China (nos. 81071720 and 81172045), Shandong Provincial Programs for Science and Technology Development (no. 2012GSF11820) and the Foundation for Outstanding Young Scientists in Shandong Province (no. 2006BS03066).

\section{References}

1. Jemal A, Siegel R, Xu J and Ward E: Cancer statistics, 2010. CA Cancer J Clin 60: 277-300, 2010.

2. Jemal A, Siegel R, Ward E, Hao Y, Xu J and Thun MJ: Cancer statistics, 2009. CA Cancer J Clin 59: 225-249, 2009.

3. Dehm SM and Tindall DJ: Androgen receptor structural and functional elements: role and regulation in prostate cancer. Mol Endocrinol 21: 2855-2863, 2007.

4. Buchanan G, Irvine RA, Coetzee GA and Tilley WD: Contribution of the androgen receptor to prostate cancer predisposition and progression. Cancer Metastasis Rev 20: 207-223, 2001.

5. Agoulnik IU and Weigel NL: Androgen receptor action in hormone-dependent and recurrent prostate cancer. J Cell Biochem 99: 362-372, 2006.

6. Taplin ME and Balk SP: Androgen receptor: a key molecule in the progression of prostate cancer to hormone independence. J Cell Biochem 91: 483-490, 2004.

7. Feldman BJ and Feldman D: The development of androgen-independent prostate cancer. Nat Rev Cancer 1: 34-45, 2001.

8. Pienta KJ and Bradley D: Mechanisms underlying the development of androgen-independent prostate cancer. Clin Cancer Res 12: 1665-1671, 2006.

9. Chen Y, Sawyers CL and Scher HI: Targeting the androgen receptor pathway in prostate cancer. Curr Opin Pharmacol 8: 440-448, 2008.

10. Knudsen KE and Scher HI: Starving the addiction: new opportunities for durable suppression of AR signaling in prostate cancer. Clin Cancer Res 15: 4792-4798, 2009.

11. Eder IE, Culig Z, Ramoner R, Thurnher M, Putz T, Nessler-Menardi C, Tiefenthaler M, Bartsch G and Klocker H: Inhibition of LncaP prostate cancer cells by means of androgen receptor antisense oligonucleotides. Cancer Gene Ther 7: 997-1007, 2000. 
12. Zegarra-Moro OL, Schmidt LJ, Huang H and Tindall DJ: Disruption of androgen receptor function inhibits proliferation of androgen-refractory prostate cancer cells. Cancer Res 62: $1008-1013,2002$

13. Wright ME, Tsai MJ and Aebersold R: Androgen receptor represses the neuroendocrine transdifferentiation process in prostate cancer cells. Mol Endocrinol 17: 1726-1737, 2003.

14. Chen CD, Welsbie DS, Tran C, et al: Molecular determinants of resistance to antiandrogen therapy. Nat Med 10: 33-39, 2004.

15. Hååg P, Bektic J, Bartsch G, Klocker H and Eder IE: Androgen receptor down regulation by small interference RNA induces cell growth inhibition in androgen sensitive as well as in androgen independent prostate cancer cells. J Steroid Biochem Mol Biol 96 251-258, 2005

16. Compagno D, Merle C, Morin A, Gilbert C, Mathieu JR, Bozec A, Mauduit C, Benahmed M and Cabon F: SIRNA-directed in vivo silencing of androgen receptor inhibits the growth of castration-resistant prostate carcinomas. PLoS One 2: e1006, 2007.

17. Di Giacomo G, Koss M, Capellini TD, Brendolan A, Pöpperl H and Selleri L: Spatio-temporal expression of Pbx3 during mouse organogenesis. Gene Expr Patterns 6: 747-757, 2006.

18. Lichtenauer UD, Duchniewicz M, Kolanczyk M, et al: Pre-B-cell transcription factor 1 and steroidogenic factor 1 synergistically regulate adrenocortical growth and steroidogenesis. Endocrinology 148: 693-704, 2007.

19. Carleton M, Cleary MA and Linsley PS: MicroRNAs and cell cycle regulation. Cell Cycle 6: 2127-2132, 2007.

20. Schmittgen TD: Regulation of microRNA processing in development, differentiation and cancer. J Cell Mol Med 12: 1811-1819, 2008.

21. Bartel DP: MicroRNAs: genomics, biogenesis, mechanism, and function. Cell 116: 281-297, 2004.

22. Ambros V: MicroRNA pathways in flies and worms: growth, death, fat, stress and timing. Cell 113: 673-676, 2003.

23. Hwang HW and Mendell JT: MicroRNAs in cell proliferation, cell death, and tumorigenesis. Br J Cancer 96 Suppl: R40-R44, 2007.

24. Wiemer EA: The role of microRNAs in cancer: no small matter Eur J Cancer 43: 1529-1544, 2007.

25. Takahashi Y, Forrest AR, Maeno E, Hashimoto T, Daub CO and Yasuda J: Mir-107 and mir-185 can induce cell cycle arrest in human non small cell lung cancer cell lines. PLoS One 4: e6677, 2009.

26. Imam JS, Buddavarapu K, Lee-Chang JS, Ganapathy S, Camosy C, Chen Y and Rao MK: MicroRNA-185 suppresses tumor growth and progression by targeting the six 1 oncogene in human cancers. Oncogene 29: 4971-4979, 2010.

27. Liu M, Lang N, Chen X, et al: MiR-185 targets RhoA and Cdc42 expression and inhibits the proliferation potential of human colorectal cells. Cancer Lett 301: 151-160, 2011.

28. Akçakaya P, Ekelund S, Kolosenko I, et al: MiR-185 and miR-133b deregulation is associated with overall survival and metastasis in colorectal cancer. Int J Oncol 39: 311-318, 2011.
29. Livak KJ and Schmittgen TD: Analysis of relative gene expression data using real-time quantitative PCR and the 2(-Delta Delta C(T)) method. Methods 25: 402-408, 2001

30. Akao Y, Nakagawa Y and Naoe T: let-7 microRNA functions as a potential growth suppressor in human colon cancer cells. Biol Pharm Bull 9: 903-906, 2006.

31. Majid S, Dar AA, Saini S, et al: miR-23b represses proto-oncogene Src kinase and functions as methylation-silenced tumor suppressor with diagnostic and prognostic significance in prostate cancer. Cancer Res 72: 6435-6446, 2012.

32. Ni Y, Meng L, Wang L, Dong W, Shen H, Wang G, Liu Q and Du J: MicroRNA-143 functions as a tumor suppressor in human esophageal squamous cell carcinoma. Gene 517: 197-204, 2013

33. Wu N, Lin X, Zhao X, Zheng L, Xiao L, Liu J, Ge L and Cao S: MiR-125b acts as an oncogene in glioblastoma cells and inhibits cell apoptosis through p53 and p38MAPK-independent pathways. Br J Cancer 109: 2853-2863, 2013.

34. Zhang B, Pan X, Cobb GP and Anderson TA: MicroRNAs as oncogenes and tumor suppressors. Dev Biol 302: 1-12, 2007.

35. Liu H, Brannon AR, Reddy AR, et al: Identifying mRNA targets of microRNA dysregulated in cancer: with application to clear cell renal cell carcinoma. BMC Syst Biol 4: 51, 2010.

36. Abaan OD and Toretsky JA: PTPI1: a large phosphatase with a split personality. Cancer Metastasis Rev 27: 205-214, 2008.

37. Mangelsdorf DJ, Thummel C, Beato M, et al: The nuclear receptor superfamily: the second decade. Cell 83: 835-839, 1995.

38. Heinlein CA and Chang C: Androgen receptor (AR) coregulators: an overview. Endocr Rev 23: 175-200, 2002.

39. Chmelar R, Buchanan G, Need EF, Tilley W and Greenberg NM: Androgen receptor coregulators and their involvement in the development and progression of prostate cancer. Int J Cancer 120: 719-733, 2007.

40. Chen S, Song CS, Lavrovsky Y, Bi B, Vellanoweth R, Chatterjee B and Roy AK: Catalytic cleavage of the androgen receptor messenger RNA and functional inhibition of androgen receptor activity by a hammerhead ribozyme. Mol Endocrinol 12: 1558-1566, 1998.

41. Eder IE, Hoffmann J, Rogatsch H, Schafer G, Zopf D, Bartsch G and Klocker H: Inhibition of $\mathrm{LNCaP}$ prostate tumor growth in vivo by an antisense oligonucleotide directed against the human androgen receptor. Cancer Gene Ther 9: 117-125, 2002.

42. Cheng H, Snoek R, Ghaidi F, Cox ME and Rennie PS: Short hairpin RNA knockdown of the androgen receptor attenuates ligand independent activation and delays tumor progression. Cancer Res 66: 10613-10620, 2006.

43. LiaoX, Tang S, Thrasher JB, Griebling TL andLi B: Small-interfering RNA-induced androgen receptor silencing leads to apoptotic cell death in prostate cancer. Mol Cancer Ther 4: 505-515, 2005.

44. Lim AC and Attard G: Improved therapeutic targeting of the androgen receptor: rational drug design improves survival in castration-resistant prostate cancer. Curr Drug Targets 14: 408-419, 2013. 